the training of recruits. Gradually, however, improvements have occurred and a group of dedicated, hard-working, and efficient contact tracers has now been established in England and Wales.

There are, however, serious problems with recruitment, training, status, pay, conditions of work, and career prospects. These problems have been highlighted by the recent reorganization of the N.H.S., in which contact tracers are now employed by the area health boards and will be seconded to the clinics for sexually transmitted diseases, where they will work under the direct supervision of the physician-in-charge.

These workers have their own society, the Society of Social Workers in Venereal Diseases, which holds an annual conference. A recent meeting of the society agreed to recommend to the Department of Health that the best name for contact tracers is "social health workers"; it also called for better standards of training and pay. Both status and remuneration have been unsatisfactory in the past. Recruits for this exacting work come from the ranks of health visitors, nurses, social workers, sociologists, and those without any formal training. The most important qualities are the personality of the recruit and her ability to form a good rapport with patients; but qualifications and training are important if a satisfactory career structure is to be developed.

A recent report of a joint subcommittee on sexually transmitted diseases of the Scottish Home and Health Department and the Scottish Health Services Council ${ }^{1}$ drew attention to the importance of contact tracing in controlling the spread of these diseases. It stressed the urgent need for recruitment and to provide a career structure and suitable in-service training programmes. Meanwhile in England research into several aspects of contact tracing is being promoted by the Health Education Council and Department of Health and Social Security. Contact tracing can be both interesting and rewarding and, provided a suitable career structure and satisfactory standards of pay can be established, the prospects for those taking up this work are excellent. The opportunity provided by reorganization of the N.H.S. to achieve these aims should not be allowed to drift by.

1 Scottish Home and Health Department, Scottish Health Services Council, Sexually Transmitted Diseases. 1974. H.M.S.O. 29p.

\section{Infection and Diabetes}

An infection in a diabetic can upset blood glucose control and lead to symptoms, even to the extent of precipitating ketosis. Infections may therefore be more of a nuisance to diabetics than to the rest of the population and cause more problems for their doctors-but are they more prone to infection? In the past they most certainly were. Before the advent of insulin therapy, infection was a major cause of death among diabetics and, among the infections, tuberculosis was the leader. As recently as the 1950s reports were still appearing of excess mortality or more florid and rapidly progressive tuberculosis among diabetics, though the disparity between them and non-diabetics was diminishing. ${ }^{1}$ However, diabetics have shared in the decline in tuberculosis in most countries.

There are certainly special circumstances affecting the diabetic which may predispose to infection. Pruritus vulvae and less often balanitis in the male are associated with candidial infections; this may possibly be due to heavy glycosuria or the increased glucose content of the secretions of the genital tract favouring the growth of the organisms. If bladder emptying is impaired by autonomic neuropathy the chronic retention of urine predisposes to urinary tract infection. Catheterization, too, is now recognized as a source of infection ${ }^{2}$; but in the absence of a history of catheterization (or other instrumentation) or autonomic neuropathy it seems that the diabetic is no more prone to urinary infection than the non-diabetic. ${ }^{34}$ The excess of pyelonephritis in post-mortem or biopsy studies of kidneys of diabetics is now regarded as evidence of vascular disease and not of infection. Necrotizing papillitis is traditionally associated with the diabetic, but is now a rare event and is more likely to be associated with abuse of phenacetin. The most common site of infection in diabetics is the feet of patients, usually elderly, with peripheral neuropathy, arterial insufficiency, or a combination of the two. ${ }^{5}$

Apart from these special circumstances, the evidence that diabetics-at least in our relatively hygienic society-are more prone to infection is fragmentary. Diabetics do who acquire infections may well present therapeutic problems because of associated loss of control or because of slower response to chemotherapy or antibiotics. Some abnormality of the natural defence against infection has long been suspected and sought in the diabetic. In general, humoral factors have not been found abnormal, but recently evidence has been found of cellular abnormalities. In 1964 Bybee and Rogers ${ }^{6}$ showed that phagocytosis was impaired in ketotic diabetics, and later Bagdade et al. made similar observations on whole blood from patients with hyperglycaemia but without ketoacidosis. This defect in phagocytosis disappeared after insulin therapy. Mowat and Baum ${ }^{8}$ reported that migration of polymorphonuclear leucocytes was delayed in the diabetic, and Bagdade et al. have now reported ${ }^{9}$ further investigations. They studied patients with poor control but without ketoacidosis, and confirmed that there was impaired phagocytosis in their serum and that this was restored towards normal with hypoglycaemic therapy. Serum from the diabetics also reduced phagocytosis by control granulocytes, while serum from controls improved phagocytosis by diabetics' granulocytes. Furthermore, dilution of diabetics' serum reduced the inhibitory effect on control granulocytes, while addition of glucose to normal serum resulted in inhibition of normal granulocytes, suggesting that hyperglycaemia itself may determine the change in granulocyte function. Certainly, glucose metabolism is important in the granulocyte ${ }^{10}$ and a few minutes after the start of phagocytosis, cell respiration suddenly increases. ${ }^{11}$

In The Doctor's Dilemma Shaw had Sir Ralph BloomfieldBonington say "There is at bottom only one genuinely scientific treatment for all diseases ... Stimulate the phagocytes." Perhaps the injunction for diabetics should be to disinhibit the phagocytes by effectively lowering the blood sugar.

1 Younger, D., and Hadley, W. B., In Foslin's Diabetes Mellitus, 11th edition. ed. A. Marble et al., Philadelphia, Lea and Febiger, 1971. Vejlsgaard, R., Acta Medica Scandinavica, 1966, 179, 173.

${ }^{3}$ Pometta, D., et al., New England fournal of Medicine, 1967, 276, 1118.

4 O'Sullivan, D. J., et al., British Medical fournal, 1961, 1, 786.

5 Catterall, R. C. F., British Fournal of Hospital Medicine, 1972, 7, 224.

Bybee, J. D., and Rogers, D. E., Fournal of Laboratory and Clinical Medicine, 1964, 64, 1 .

7 Bagdade, J. D., Nielson, K. L., and Bulger, R. J., American fournal of Medical Sciences, 1972, 263, 451.

8 Mowat, A. G., and Baum, J., New England fournal of Medicine, 1971 284, 621 .

9 Bagdade, J. D., Root, R. K., and Bulger, R. J., Diabetes, 1974, 23, 9.

10 Baehner, R. L., and Nathan, D. G., Science, 1967, 155, 835.

11 Klebanoff, S. J., Annual Review of Medicine, 1971, 22, 39. 